Canadian

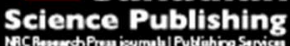

Applied Physiology, Nutrition, and Metabolism Physiologie appliquée, nutrition et métabolisme

\title{
Shrimp Oil Extracted from the Shrimp Processing Waste Reduces the Development of Insulin Resistance and Metabolic Phenotypes in Diet-induced Obese Rats
}

\begin{tabular}{|r|l|}
\hline Journal: & Applied Physiology, Nutrition, and Metabolism \\
\hline Manuscript ID & apnm-2016-0644.R1 \\
\hline Manuscript Type: & Article \\
\hline Complete List of Authors: & $\begin{array}{l}\text { Nair, Sandhya; Coastal Zones Research Institute Inc; National Research } \\
\text { Council of Canada } \\
\text { Gagnon, Jacque; Coastal Zones Research Institute Inc } \\
\text { Pelletier, Claude.; Coastal Zones Research Institute Inc } \\
\text { Tchoukanova, Nadia; Coastal Zones Research Institute Inc } \\
\text { Zhang, Junzeng; National Research Council of Canada } \\
\text { Ewart, H. Stephen; Novaceutics Consulting } \\
\text { Ewart, Vanya; Dalhousie University } \\
\text { Jiao, Guangling; National Research Council Canada } \\
\text { Wang, Yanwen; National Research Council Canada, }\end{array}$ \\
\hline Keyword: & $\begin{array}{l}\text { Diet-induced obesity, glucose tolerance, insulin sensitivity, oxidative stress, } \\
\text { inflammation, rat }\end{array}$ \\
\hline
\end{tabular}




\section{Shrimp Oil Extracted from the Shrimp Processing Waste Reduces the Development of Insulin Resistance and Metabolic Phenotypes in Diet-induced Obese Rats}

Sandhya Nair ${ }^{\mathrm{a}, \mathrm{b}}$ Jacques Gagnon ${ }^{\mathrm{a},}$, Claude Pelletier ${ }^{\mathrm{a}}$, Nadia Tchoukanova ${ }^{\mathrm{a}}$, Junzeng Zhang ${ }^{\mathrm{c}}$, H. Stephen Ewart ${ }^{\mathrm{d}}$, Vanya Ewart ${ }^{\mathrm{e}}$, Guangling Jiao ${ }^{\mathrm{a}, \mathrm{c}}$, and Yanwen Wang ${ }^{\mathrm{b}, *}$

${ }^{a}$ Coastal Zones Research Institute Inc (CZRI), Shippagan, NB , Canada

${ }^{b}$ Natural Health Products Program, Aquatic and Crop Resource Development, National Research Council of Canada, Charlottetown, PE, Canada

${ }^{c}$ Natural Health Products Program, Aquatic and Crop Resource Development, National Research Council of Canada, Halifax, NS, Canada

${ }^{\mathrm{d}}$ Novaceutics Consulting, Halifax, NS, Canada

${ }^{\mathrm{e}}$ Department of Biochemistry \& Molecular Biology, Dalhousie University, Halifax, NS, Canada

Running title: Shrimp oil and insulin resistance in rats

Correspondence:

Yanwen Wang, PhD. Aquatic and Crop Resource Development, National Research Council of Canada, 550 University Avenue, Charlottetown, PE, Canada C1A 4P3. Tel: (902) 566-7953. Email: yanwen.wang@nrc.ca

Jacques Gagnon, PhD. Coastal Zones Research Institute Inc (CZRI), Shippagan, NB, Canada E8S 1J2. Tel: 506-336-6600. Email: Jacques.gagnon@umoncton.ca. 


\begin{abstract}
Abbreviations
AUC, area under the curve; CRP, C-reactive protein; DHA, docosahexaenoic acid; EPA, eicosapentaenoic acid; GPx, glutathione peroxidase; GR, glutathione reductase; GSH, reduced glutathione; GSSG, oxidized glutathione; HOMA-IR, homeostatic model assessment of insulin resistance; HFC, high-fat control; HDL-C, high-density lipoprotein cholesterol; IL-6, interleukin6; IL-1 $\beta$, interleukin-1 $\beta$; ITT, insulin tolerance test; LFC, low-fat control; MCP-1, monocyte chemoattractant protein-1; MUFA, monounsaturated fatty acids, OGTT, oral glucose tolerance test; PUFA, polyunsaturated fatty acids; SO10, HFC diet with $10 \%$ of lard being replaced with shrimp oil; SO15, HFC diet with $15 \%$ of lard being replaced with shrimp oil; SO20, HFC diet with $20 \%$ of lard being replaced with shrimp oil; SOD, superoxide dismutase; TAG, triacylglycerols; TBARS, thiobarbituric acid reactive substances; T-C, total cholesterol; TNF- $\alpha$, tumor necrosis factor- $\alpha$.
\end{abstract}




\begin{abstract}
Diet-induced obesity, insulin resistance, impaired glucose tolerance, chronic inflammation and oxidative stress represent the main features of type 2 diabetes mellitus. The present study was conducted to examine the efficacy and mechanisms of shrimp oil on glucose homeostasis in obese rats. Male CD rats fed a high-fat diet (60 kcal\% fat) and 20\% fructose-drinking water were divided into four groups and treated with the dietary replacement of $0,10 \%(\mathrm{SO} 10), 15 \%$ (SO15), or 20\% (SO20) of lard with shrimp oil for 10 weeks. Age-matched rats fed a low-fat diet (10 kcal\% fat) were used as the normal control. Rats on the high-fat diet showed impaired $(\mathrm{p}<$ 0.05) glucose tolerance and insulin resistance compared with rats fed the low-fat diet. Shrimp oil improved $(\mathrm{p}<0.05)$ oral glucose tolerance, insulin response and homeostatic model assessmentestimated insulin resistance index, decreased serum insulin, leptin and hemoglobin A1c and free fatty acids, and increased adiponectin. Shrimp oil also increased $(\mathrm{p}<0.05)$ antioxidant capacity and reduced oxidative stress and chronic inflammation. The results demonstrated that shrimp oil dose-dependently improved glycemic control in obese rats through multiple mechanisms.
\end{abstract}

Keywords: Diet-induced obesity, glucose tolerance, insulin sensitivity, oxidative stress, inflammation, rat 


\section{Introduction}

Obesity is growing in high income and low to middle-income countries alike, particularly in urban settings (Popkin et al., 2012). As the rates of obesity continue to rise, insulin resistance is becoming one of society's major public health issues. Obesity is considered to be a result of an imbalance between calories consumed and calories expended (Hill et al., 2012). Tissues such as muscle, fat and liver become less responsive to insulin and results in a state of insulin resistance; this is characterized by an impaired ability of insulin to inhibit glucose output from the liver and to promote glucose uptake in the peripheral tissues, mainly muscle and adipose tissues (Wilcox, 2005). Although increasing physical activity improves weight loss (Jakicic, 2009) and insulin sensitivity (Ross, 2003), it is difficult to achieve in modern societies. Alternatively, dietary modifications including taking supplements and nutraceuticals may improve insulin sensitivity and lower the incidence of type 2 diabetes and other metabolic diseases (Mirmiran et al., 2014).

Among different foods and nutraceuticals, seafood appears to have an inverse relationship with the incidence of metabolic syndrome (Baik et al., 2010; Kim et al., 2015; Patel et al., 2009). Fish is mostly studied seafood for its various nutritional values and health benefits (Hosomi et al., 2012). Supplementation of fish oil is reported to improve insulin resistance (Albert et al., 2014; Lombardo and Chicco, 2006), an effect that might be due to its content of monounsaturated fatty acids (MUFA) and n-3 polyunsaturated fatty acids (PUFA) compared to other animal foods (Larsen et al., 2011; Nettleton and Katz, 2005; Ramel et al., 2008; Swanson et al., 2012).

Northern shrimp (Pandalus borealis) is the most abundant cold-water shrimp in the North Atlantic and Pacific areas and has been widely harvested since the early 1960s. The head and tail 
of this shrimp is rich in lipids. A recent study showed that Northern shrimp oil contains not only a high content of n-3 PUFA but also a high concentration of antioxidants (Subramanian et al., 2015), the latter are also beneficial to metabolic health and glucose homeostasis (Vincent et al., 2009). However, the heads and tails of shrimp are disposed as a waste material at shrimp processing plants, impacting the environment and increasing production cost. The reason that shrimp heads and tails are discarded as waste by the processing industry is the lack of understanding regarding their nutritional value and potential for value-added products. As a result, the industry has not invested in technology and equipment required to efficiently extract valuable ingredients such as n-3 PUFAs and antioxidants from the waste stream. With our recent development of an extraction technology (US 20160145533 A1), efficient extraction and preservation of oil from shrimp processing wastes has become feasible. The present study reports that PUFA-rich oil extracted from the shrimp processing waste with this technology has beneficial effects on insulin sensitivity, glycemic control, and other metabolic phenotypes associated with diabetes in a rat model of insulin resistance.

\section{Methods and Materials}

\subsection{Preparation of shrimp oil for animal study and analyses}

The shrimp oil used for this study was extracted from the processing water of Northern shrimps caught in the Atlantic area, specifically the Anticosti fishing zone of St-Lawrence Gulf and further cleaned using a patented technology (US 20160145533 A1). Briefly, during the processing of Northern shrimp the water was recovered and subjected to a dissolved air flotation system with a flocculating agent. The suspended and/or dissolved solids formed aggregates, which were collected and directed into a horizontal centrifuge to further separate 
solids from liquids. The liquid phase containing mainly water and oil was pumped into a 3phase vertical centrifuge to separate oil from water and solid residues. The oil fraction was used for animal studies.

After washing with $5 \mathrm{~mL}$ hexane, a Supelclean ${ }^{\mathrm{TM}}$ LC-Si SPE cartridge (Sigma-Aldrich Corp., St Louis, MO, USA) was loaded with $10 \mathrm{~mL}$ of shrimp oil dissolved in $5 \mathrm{~mL}$ hexane. The cartridge was eluted with $2 \times 5 \mathrm{~mL}$ hexane containing $6 \%$ diethyl ether to obtain a triacylglycerol-rich fraction and then $2 \times 5 \mathrm{~mL}$ acetone containing $200 \mathrm{mg} / \mathrm{L}$ BHT to obtain the pigment fraction. The two acetone fractions were pooled, evaporated under nitrogen and stored at $-20{ }^{\circ} \mathrm{C}$ until analysis.

\subsection{Analysis of astaxanthin in shrimp oil}

The shrimp oil was subjected to a solid phase extraction to separate sterols, carotenoids and other components using silica Strat-si-1 cartridge (Phenomenex Inc., Torrance, CA). The astaxanthin was released from the mono and di-esters following the reported method (Del Campo et al., 2004) and analyzed on an Varian Prostar 240 HPLC (Agilent Technologies, Mississauga, ON) according to the method described elsewhere (Yuan and Chen, 1997).

\subsection{Analysis of vitamins $A$ and $E$ in shrimp oil}

The content of vitamin A in shrimp oil was measured according to an AOAC (Association of Official Analytical Chemists) method (AOAC 2001.13-2001) while the content of vitamin E was determined using an AACC (American Association of Clinical Chemistry) method (AACC 8606) using the HPLC. 


\subsection{Analysis of fatty acids in shrimp oil}

An aliquot of shrimp oil was transferred to a screw-capped glass tube that contains $1 \mathrm{mg}$ of nonadecanoic acid (C19:0) as an internal standard and evaporated to dryness under nitrogen. Fatty acids were trans-esterified by re-dissolving the dried lipid samples in $3 \mathrm{~mL}$ of methanol/toluene mix $(3: 2, \mathrm{v} / \mathrm{v})$ with additional $2 \mathrm{ml}$ of $5 \%$ acetyl chloride in methanol. The tubes were flushed with nitrogen, closed tightly and heated at $100^{\circ} \mathrm{C}$ for $1 \mathrm{hr}$, with gentle shaking every $15 \mathrm{~min}$. After cooling to room temperature, $4 \mathrm{~mL}$ of distilled water and $1 \mathrm{~mL}$ of hexane were added. The tubes were shaken vigorously and centrifuged at $2,000 \mathrm{rpm}$ for $2 \mathrm{~min}$. The hexane/toluene phase containing methylated fatty acids was transferred to a GC vial. An Agilent DB-WAX capillary column $(20 \mathrm{~m}$ x $0.18 \mathrm{~mm}$ ID x $0.18 \mu \mathrm{m})$ on an Agilent $7890 \mathrm{~B}$ GC (Agilent Technologies, Mississauga, ON), equipped with an autosampler, a purge ultimate union backflush and flame ionization detector, was used to separate and quantify the fatty acid methyl esters. One $\mu \mathrm{L}$ of sample was injected onto the column using a split mode (1:50) and hydrogen was used as the carrier gas. The initial column/oven temperature was set at $140^{\circ} \mathrm{C}$ for $0.2 \mathrm{~min}$, increased to $170^{\circ} \mathrm{C}$ at $40^{\circ} \mathrm{C} / \mathrm{min}$ and held for $3 \mathrm{~min}$, then to $185^{\circ} \mathrm{C}$ at $4.9^{\circ} \mathrm{C} / \mathrm{min}$, further to $200^{\circ} \mathrm{C}$ at $2.5^{\circ} \mathrm{C} / \mathrm{min}$ and held $3 \mathrm{~min}$, and finally ramped to $220^{\circ} \mathrm{C}$ at $2.5^{\circ} \mathrm{C} / \mathrm{min}$. At the end of each run, the purge ultimate union backflush was used to flush out the sterols and other less volatile compounds through the split line of the injection port. Individual fatty acids were identified against the retention time of corresponding standards running in parallel with the samples, and quantitated in reference to the internal standard.

\subsection{Rats and diets}


The animal use and experimental procedures were approved by the Joint Animal Care and Research Ethics Committee of the National Research Council Canada-Institute for Nutrisciences and Health and the University of Prince Edward Island, Canada. The study was conducted in accordance with the guidelines of the Canadian Council on Animal Care.

Male CD rats (200-225 g) were obtained from Charles River (Saint Constant, QC, Canada) and housed individually in rat cages with a 12-hr light:dark cycle and free access to rodent chow and water. After 7 days of adaption, body weight was obtained and blood glucose was measured. Rats were then randomly assigned based on body weight and blood glucose concentration into two groups of 12 rats and 48 rats, respectively. The group of 12 rats was fed a low-fat control (LFC) diet and the remaining 48 rats were fed a high-fat control (HFC) diet. A week later, rats on the HFC diet were segregated into four groups. One group was used as the control (continued on the regular HFC diet) whereas the other three groups were fed the HFC diet with 10\% (SO10), $15 \%$ (SO15), or 20\% (SO20) of lard being replaced with shrimp oil. The composition of diets is provided in Supplementary table S1. Rats on the HFC, SO10, SO15 and SO20 diets also received $20 \%$ fructose-drinking water, which was started at the same time as the HFC diet and prepared every other day. The inclusion of $20 \%$ fructose in drinking water in addition to the high-fat diet was used to accelerate the development of insulin resistance (Stranahan et al., 2008). Rats were weighed weekly and food intake was monitored daily for 10 weeks. At the end of the study, the rats were fasted overnight and then anesthetized by inhalation of isoflurane (Pharmaceutical Partners of Canada Inc. Richmond Hill, ON, Canada) and blood was collected immediately via cardiac puncture into serum and plasma tubes (BD Biosciences, ON, Canada). The rats were euthanized by cervical dislocation and the liver was immediately dissected, 
weighed, and flash-frozen in liquid nitrogen. Serum, plasma and liver samples were stored in a $80^{\circ} \mathrm{C}$ freezer.

\subsection{Glucose and insulin tolerance tests}

Oral glucose tolerance test (OGTT) was carried out during weeks 4 and 9 of the treatment and insulin tolerance test (ITT) was conducted in all rats during week 10 of treatment after 4-hr fasting as reported previously (Wang et al., 2015). The area under the curve (AUC) of blood glucose levels was calculated for the OGTT and ITT, respectively.

\subsection{Analytical of blood glucose and lipids}

The fasting serum glucose concentration was measured in triplicate as reported previously (Wang et al., 2015). The fasting serum total cholesterol (T-C), HDL cholesterol (HDL-C) and triacylglycerol (TAG) were measured in triplicate following the published methods (Wang et al., 2014). The serum free fatty acid was determined in duplicate using commercial kits following the kit instructions (Abcam Inc., Toronto, ON, Canada).

\subsection{Measurement of serum insulin, leptin, adiponectin, and HbAlc}

Glycated hemoglobin (HbA1c) in the fasting serum was measured in duplicate using a commercial assay kit (Crystal Chem, IL, USA) following the kit instructions. The concentration of serum insulin, leptin, and adiponectin was also measured using the ELISA kits, with the rat insulin kits being obtained from Mercodia (Uppsala, Sweden), leptin kits from R\&D Systems (Minneapolis, MN, USA) and adiponectin kits from Abcam Inc. (Toronto, On, Canada). The coefficient of variance between replicates was $7.2 \%, 5.1 \%$, and $4.3 \%$ and detection range was 
1.56-100 ng/ml, 0.15-5.5 $\mu \mathrm{g} / \mathrm{ml}$, and 62.5-4,000 pg/ml for adiponectin, insulin and leptin ELISA kits, respectively. All samples were within the detection range except those for adiponectin for which a dilution factor of 1:400 was applied.

\subsection{Measurement of inflammatory biomarkers}

The serum concentrations of C-reactive protein (CRP), tumor necrosis factor- $\alpha$ (TNF- $\alpha$ ), interleukin-6 (IL-6), interleukin-1 $\beta$ (IL-1 $\beta$ ), and monocyte chemoattractant protein-1 (MCP-1) were measured in duplicate using commercial ELISA kits following the kits instructions. The rat CRP ELISA kit was from Abcam Inc. (Toronto, ON, Canada), the TNF- $\alpha$ kit from Biolegend (San Diego, CA, USA), the IL-1 $\beta$ and MCP-1 ELISA kits from Affymetrix-eBioscience (San Diego, CA, USA), and the IL-6 ELISA kit from Cusabio (Wuhan, Hubei, China). The coefficient of variation between replicates was $7.4 \%, 6.7 \%,<10 \%,<8 \%$, and $<4.9 \%$ for TNF- $\alpha$, MCP- 1 , IL-1 $\beta$, IL-6, and CRP assays, respectively. The detection range was 7.5-800 pg/ml, 0.31-20 $\mathrm{pg} / \mathrm{ml}$, and $0.78-50 \mathrm{ng} / \mathrm{ml}$ for the TNF- $\alpha$, IL-6 and CRP, respectively. The sensitivity was $>4.7$ $\mathrm{pg} / \mathrm{ml}$ and $>4 \mathrm{pg} / \mathrm{ml}$ for the MCP-1 and IL-1 $\beta$ assays, respectively. The samples were diluted at 1:60,000 for CRP analysis.

\subsection{Measurement of oxidative stress biomarkers}

The activity of catalase, superoxide dismutase (SOD), glutathione peroxidase (PGx), glutathione reductase (GR), reduced glutathione (GSH), oxidized glutathione (GSSG), and thiobarbituric acid reactive substances (TBARS) in the liver and the concentration of total antioxidants in the serum (1:20 dilution) were measured in duplicate using the commercial assay kits (Cayman chemicals, MI, USA) following the kits instructions. It should be noted that TBARS assay is 
non-specific, measuring several products of oxidative stress rather than directly measure reactive oxygen species, which have extremely short half-lives (Pryor, 1991).

\subsection{Calculation of homeostatic model assessment of insulin resistance}

The homeostatic model assessment of insulin resistance (HOMA-IR) was calculated as (glucose in $\mathrm{mmol} / \mathrm{L} \times$ insulin in $\mathrm{mU} / \mathrm{L}) / 22.5$.

\subsection{Statistical Analysis}

All analyses were performed using SAS software version 9.2 (SAS Institute, North Carolina, USA). The difference between the HFC and LFC was analyzed using the Student's $t$-test. The treatment effect was analysed using one-way ANOVA. Repeated measures t-test and repeated measures one-way ANOVA were employed for the parameters that were measured multiple times. When a significant treatment effect was obtained, differences among the HFC, SO10, SO15 and SO20 were determined by using the least squares means test adjusted to Tukey. Significance level was set at $\mathrm{p}<0.05$. The results are presented as means \pm S.E.M $(n=11-12)$.

\section{Results}

\subsection{Composition of shrimp oil}

The shrimp oil used in the present study contains $\geq 95 \%$ of triacylglycerols and $1.7 \%$ sterols (Subramanian et al., 2015). Although not measured in the present study, the phospholipids content was estimated to be less than $3 \%$ considering that the oil also contains a small amount of free fatty acids and other fat-soluble components. Fatty acid composition and content of shrimp oil are provided in Supplementary table S2. It was found that this oil contained a low content of 
saturated fatty acids (18.2\%) while having high levels of MUFA (54.6\%) and PUFA (27.2\%). Moreover, there were many different MUFA with different positions of the first double-bond. The predominant MUFA were C18:1n-9 (13.0\%), C16:1n-7 (12.0\%), C22:1n-11+13 (9.7\%), C20:1n-9 (7.5\%), C18:1n-7 (4.1\%), C22:1n-9 (2.6\%), and C20:1n-7 (2.3\%). A high level of n-3 PUFA, especially EPA (9.9\%) and DHA (10.3\%), was another beneficial characteristic of this oil. Thus, the fatty acid composition of the experimental diets differed substantially. The saturated fatty acids decreased whereas the monounsaturated and polyunsaturated fatty acids increased with the replacement of $10-20 \%$ dietary lard with shrimp oil. There was a marked increase of n-3, especially EPA and DHA, while n-6 fatty acids were decreased by replacing lard with shrimp oil (Supplementary table S3). In addition to its fatty acid profile, shrimp oil used in the present study was also analyzed for the content of antioxidants (Supplementary table S4). Ketocarotenoid astaxanthin $(\geq 400 \mu \mathrm{g} / \mathrm{g})$, vitamin $\mathrm{E}(\geq 50 \mathrm{IU} / 100 \mathrm{~g})$ and vitamin $\mathrm{A}(\geq 1000$ $\mathrm{RE} / 100 \mathrm{~g}$ ) were the main antioxidants. Furthermore, astaxanthin in this shrimp oil existed as $75 \%$ diesters and 23\% monoesters and of the total astaxanthin, 57\% was trans-astaxanthin and 43\% was cis-astaxanthin (Subramanian et al., 2015). In addition, the oil contained 1.7\% sterols and of which $0.8 \%$ was cholesterol (Subramanian et al., 2015). Analysis for contaminants in the shrimp oil revealed that dioxins, furans and polychlorinated biphenyls were below the levels of detection or the acceptable limits for consumption set by regulatory agencies such as the Canadian Food Inspection Agency (data not shown).

\subsection{Body weight and food intake}

As shown in Table 1, the HFC group had higher $(\mathrm{p}<0.05)$ body weights than the LFC group. However, no differences were found among the HFC, SO10, SO15 and SO20 groups. The food 
intake was lower $(\mathrm{p}<0.05)$ in the HFC group than in the LFC group (Table 2). The shrimp oil supplementation did not alter food consumption from week 1 to week 9 of the treatment. On week 10, food intake increased in the HFC group whereas it decreased in the SO10, SO15 and SO20 groups $(\mathrm{p}<0.01)$.

\subsection{Shrimp oil improves oral glucose tolerance}

Semi-fasting (4-hr) blood glucose was elevated in the HFC group as compared to the LFC group from week 7 to the end of the study (Table 3). The three doses of shrimp oil did not show significant effects compared to the HFC group. The fasting (12-hr) serum glucose levels did not differ among the HFC, LFC and treatment groups (Table 4).

The oral glucose loading induced significant increases of blood glucose level in rats of the HFC group at every time point post oral glucose loading compared to the LFC control in weeks 4 (Figure 1A) and 9 (Figure 1B) of the treatment, respectively, indicating that glucose intolerance was developed in the HFC group as a result of free access to the high-fat diet and $20 \%$ fructosedrinking water. This effect was reversed completely or partially by replacing a small portion of dietary lard with shrimp oil. In the first oral glucose tolerance test conducted during week 4 of the treatment, rats of the SO20 group showed lower $(\mathrm{p}<0.05)$ blood glucose levels at 90 and $120 \mathrm{~min}$ after the oral glucose loading (Figure 1A). The same test was repeated in week 9 of the treatment. The blood glucose levels was lowered $(\mathrm{p}<0.05)$ in both the SO20 and SO15 groups at 60, 90, and 120 min (Figure 1B) and the SO20 group also showed lower $(\mathrm{p}<0.05)$ glucose levels at 30 min compared to the HFC group. Despite a lack of an effect on body weight, replacing $20 \%$ or $15 \%$ of dietary lard by shrimp oil resulted in a significant improvement of oral 
glucose tolerance. Consistently, the AUC of OGTT in either week 4 or week 9 of the treatment showed significant benefits of shrimp oil (Table 5). The AUC was higher $(\mathrm{p}<0.01)$ in the HFC group than in the LFC group in both OGTTs. It was reduced $(\mathrm{p}<0.01)$ by $7 \%$ in the SO20 group in week 4 of treatment. A better effect was seen in week 9 of treatment, with a $12 \%$ reduction (p $<0.001)$ being observed in the SO20 group relative to the HFC group. In this test, the SO15 also showed a significant effect and $11 \%$ lower $(\mathrm{p}<0.01)$ than the HFC group.

\subsection{Shrimp oil improves insulin sensitivity}

Further to the oral glucose tolerance test, an insulin tolerance test was performed to determine the effect of shrimp oil on insulin sensitivity. Rats of the HFC group showed higher $(\mathrm{p}<0.05)$ levels of blood glucose at 0, 30, 60, 90, and $120 \mathrm{~min}$, respectively, than those of the LFC group after the intraperitoneal insulin injection (Figure 2), suggesting that rats fed the high-fat diet developed insulin resistance. Interestingly, when dietary lard was partially replaced by shrimp oil the body response to the injected insulin was significantly improved. As a result, rats in the SO20 group showed markedly lower $(\mathrm{p}<0.05)$ glucose levels at all time points except 30 min post insulin injection. The blood glucose levels were also lowered $(\mathrm{p}<0.05)$ in the SO15 group at 60, 90, and $120 \mathrm{~min}$ and in the SO10 group at 90 and 120 min compared to the HFC group. Consistently, the HFC group had a higher $(\mathrm{p}<0.001)$ AUC than the LFC group (Table 5). The shrimp oil dose-dependently decreased the AUC, which was $11 \%(\mathrm{p}<0.01), 14 \%(\mathrm{p}<0.001)$, and $17 \%(\mathrm{p}<0.0001)$ lower in the SO10, SO15 and SO20 groups, respectively, compared to the HFC group. 


\subsection{Effect of shrimp oil on serum glycated hemoglobin, insulin, leptin, and adiponectin concentrations and HOMA-IR}

The serum level of HbAlc is an important indicator of impaired glucose metabolism or insulin resistance. The HFC group markedly increased $(\mathrm{p}<0.05)$ the serum HbA1c levels compared to LFC group (Table 4). Strikingly, the elevation of HbA1c in the HFC group was reversed by replacing dietary lard with shrimp oil at the dose of 10,15 , or $20 \%$. Similarly, the serum concentrations of insulin and leptin were elevated $(\mathrm{p}<0.01)$ in the HFC group compared to that in the LFC group, whereas they were dose-dependently lowered in the SO10, SO15, and SO20 groups, respectively. Serum insulin was lower $(\mathrm{p}<0.01)$ in the SO20 group as was serum leptin $(p<0.05)$ relative to the HFC group. In contrast, the serum adiponectin was decreased by $40 \%(p$ $<0.001$ ) in the HFC group compared to the LFC group, which was reversed by the replacement of dietary lard with shrimp oil. Compared to the HFC group there were $43 \%, 52 \%$, and $60 \%$ increases $(\mathrm{p}<0.01)$ of serum adiponectin concentrations in the SO10, SO15 and SO20 groups, respectively. The HOMA-IR is calculated based on the fasting blood glucose and insulin concentrations and widely used to assess the severity of insulin resistance. The present study showed a higher $(\mathrm{p}<0.001)$ HOMA-IR index in the HFC group than the LFC group. This effect was improved by shrimp oil in a dose-dependent manner, resulting in a significantly lower $(\mathrm{p}<$ $0.05)$ value in the SO20 group than the HFC group.

\subsection{Shrimp oil improved serum lipid profile}

The replacement of dietary lard with shrimp oil dose-dependently lowered the serum total cholesterol and a significant difference was observed between the SO20 and HFC groups (p < 0.05) although no difference was observed between the HFC and LFC groups (Table 4). The 
HFC group showed lower serum TAG $(p<0.001)$ and HDL-C $(p<0.0001)$ levels than the LFC group and no significant treatment effects were observed. Compared to the LFC diet, the HFC diet increased $(\mathrm{p}<0.05)$ the serum free fatty acid concentration. This was reduced by $11 \%, 26 \%$, and $43 \%$ in the SO10, SO15, and SO20, respectively, but the changes did not reach statistical significance ( $\mathrm{p}=0.054$ for the $\mathrm{SO} 20$ versus HFC group).

\subsection{Effect of shrimp oil on total antioxidant capacity and oxidative stress biomarkers}

The serum total antioxidant capacity was not different between the HFC and LFC groups (Table 6); however, it was increased by dietary shrimp oil in a dose-dependent manner. As a result, the SO20 group showed a significantly higher value than the HFC group $(p<0.05)$. Although no differences were noticed between the HFC and LFC groups, the activity of liver glutathione reductase, catalase and SOD was increased $(\mathrm{p}<0.05)$ in the SO20 group compared to the HFC group. Moreover, the three doses of shrimp oil increased $(p<0.05)$ liver glutathione compared to the HFC group while no difference was seen between the HFC and LFC groups.

\subsection{Effect of shrimp oil on serum inflammatory biomarkers}

As shown in Table 6, TNF- $\alpha$ was increased $(\mathrm{p}<0.0001)$ by 8 -fold in the HFC group relative to the LFC group but decreased $(\mathrm{p}<0.001)$ significantly and dose-dependently by shrimp oil. A reduction of $34 \%, 36 \%$ and $71 \%$ was observed in the SO10, SO15 and SO20 groups, respectively. Shrimp oil did not show significant effects on serum MCP-1, IL-1 $\beta$, IL-6, and CRP levels.

\section{Discussion}


The present study demonstrated that rats fed the high-fat diet and fructose-drinking water gained more weight and became obese as compared with those given the low-fat diet, developed glucose intolerance and insulin and leptin resistance, and increased production of pro-inflammatory cytokine TNF- $\alpha$. These observations are in agreement with previous reports (Geng et al., 2016; Huang et al., 2004). Interestingly, these effects were reversed or significantly improved by replacing up to $20 \%$ of lard in the diet with shrimp oil. As the total fat content in each diet was identical, the improvements in glucose intolerance and insulin and leptin resistance are considered to be a result of the components of shrimp oil that are different from lard.

The improvement of oral glucose intolerance by shrimp oil was demonstrated by the results of two separate oral glucose tolerance tests, which are commonly used in both animal and human studies to evaluate glucose tolerance (Wang et al., 2015). The insulin resistance test further demonstrated that the impaired oral glucose tolerance was, at least in part, a result of a reduced response to insulin. This notion was supported by HOMA-IR index, which has been increasingly used to assess insulin resistance (Matthews et al., 1985; Singh and Saxena, 2010). This index was substantially elevated in rats fed the high-fat diet but improved with shrimp oil in a dosedependent manner, becoming normal when $20 \%$ lard was replaced with shrimp oil. Blood concentrations of insulin and leptin are important biomarkers of obesity and diabetes and vice versa (Considine et al., 1996; Koch et al., 2014; Wilcox, 2005). Rats on the HFC diet and fructose drinking water had blood insulin and leptin levels twice as much as those on the LFC diet, a hallmark of early stage insulin resistance. The significant reductions of insulin and leptin by $20 \%$ replacement of dietary lard for shrimp oil demonstrated again the beneficial effect of shrimp oil on insulin and leptin resistance. These results collectively demonstrated that replacing 
dietary lard with shrimp oil up to $20 \%$ produced significant benefits on insulin function and glycemic control.

Adiponectin mediates insulin function and glucose homeostasis and the circulating levels of adiponectin are negatively associated with body fat mass and insulin resistance (Jung and Choi, 2014; Yamauchi et al., 2001). In line with previous reports, the serum concentration of adiponectin was markedly reduced in rats given the high-fat diet and fructose-drinking water but this effect was abolished by replacing up to $20 \%$ of dietary lard with shrimp oil in a dosedependent manner. Adiponectin stimulates fatty acid oxidation by a sequential activation of adenosine monophosphate-activated protein kinase, P38 mitogen-activated protein kinase and peroxisome proliferator-activated receptor alpha (Yoon et al., 2006), leading to the reduction of the circulating free fatty acids. Therefore, the blood concentrations of adiponectin and free fatty acids change reciprocally as observed in the present study.

HbA1c is another well-established biomarker of insulin resistance and widely used in the diagnosis of diabetes and evaluating the effectiveness of therapies for diabetes (Lee et al., 2014; Lorenzo et al., 2010; Singh and Saxena, 2010). The blood concentration of HbA1c increases in humans and animals with insulin resistance and diabetes and decreases with improvement of disease conditions (Konda et al., 2014; Li et al., 2016; Yanni et al., 2016). The dose-dependent reductions of the serum HbA1c by replacing dietary lard with shrimp oil further demonstrated the protective effect of shrimp oil on the development of insulin resistance in diet-induced obese rats. 
The antidiabetic benefits of shrimp oil might be attributed to its multiple bioactive components. First, shrimp oil had significant amounts of MUFA and n-3 PUFA, which have been reported to be beneficial on glycemic control in rodents and humans (Albert et al., 2014; Madigan et al., 2000; Robbez Masson et al., 2008; Samimi et al., 2015). Replacing dietary saturated fatty acids with MUFA (Riserus et al., 2009) or n-6 linoleic acid with oleic acid (Madigan et al., 2000) improves insulin sensitivity. Dietary supplementation of n-3 PUFA, in particular EPA and DHA, increases the serum concentration of adiponectin in humans and animals (Gray et al., 2013; Sabour et al., 2015). Recent reports demonstrated that replacing saturated fatty acids with n-3 PUFA lowers blood glucose and HbA1c levels, HOMA-IR index, and insulin secretion in humans (Imamura et al., 2016; Iwasaki et al., 2012). Second, astaxanthin, vitamins E and vitamin A in shrimp oil increase antioxidant capacity and reduce oxidative stress (Sadighara et al., 2014). High-fat, high-fructose consumption decreases the body total antioxidant capacity, along with the inactivation of catalase and glutathione peroxidase, leading to an increase of hydrogen peroxide levels that in turn inactivate superoxide dismutase and increase superoxide anions (Savini et al., 2013). Antioxidants quench free radicals, which otherwise cause lipid peroxidation and oxidative stress and consequently the peripheral tissue insulin resistance and damage of pancreatic $\beta$-cells (Meydani et al., 1991). Astaxanthin is a strong antioxidant (Ambati et al., 2014) and a product of astaxanthin purified from shrimp is reported to lower malondialdehyde levels, enhance the activities of superoxide dismutase, catalase, glutathione peroxidase, and glutathione reductase, and increase the ratio of GSH to GSSG in alloxan-induced diabetic rats (Sila et al., 2015). Similar effects were observed in the present study. Vitamins A and E are decreased in diabetes (Valdes-Ramos et al., 2015), and accordingly the supplementation of shrimp oil may improve the status of these two vitamins. 
The anti-inflammatory effects of shrimp oil might have provided an additional protection against the development of insulin resistance as chronic inflammation is well-known to be related to obesity and diabetes (Wellen and Hotamisligil, 2005). Excess fat storage and acute hyperglycemia release pro-inflammatory cytokines into the circulating system, leading to increases of chronic inflammation in the peripheral tissues and development of insulin resistance (Johnson et al., 2012). The consumption of the high-fat diet and high-fructose drinking water induced a dramatic increase of the serum concentration of TNF- $\alpha$ that was reversed dosedependently by dietary shrimp oil. This may be attributed to the inhibitory effects of shrimp oil n-3 PUFAs (Siriwardhana et al., 2012; Trebble et al., 2003) and antioxidants (Cordero-Herrera et al., 2015; Fassett and Coombes, 2011; Rizvi et al., 2014) on chronic inflammation.

The increased serum total cholesterol level in the HFC rats was consistent with the earlier studies (Akiyama et al., 1996; Nair et al., 2014), indicating that lipid metabolism was altered by high fat and/or fructose intake. The reduction of serum total cholesterol was probably a result of bile acids sequestration and subsequent excretion from the body (Smit et al., 1991). Furthermore, shrimp oil contained a lower concentration of saturated fatty acids, especially palmitic acid, which promotes synthesis of cholesterol (Fernandez and West, 2005).

The anti-diabetic effect of shrimp oil observed in male rats is anticipated in females (Ohta et al., 2014). However, sex differences were observed in the progression and extent of insulin resistance and diabetes (Mauvais-Jarvis, 2015). Strain differences also exist, for example, the metabolic effects caused by a high-fat diet seemed to be more pronounced in Wistar than in SD 
rats (Marques et al., 2016). Another consideration is the amount of shrimp oil required to see similar effects in humans. According to the most recently published method of dose translation from rodents to humans (Nair and Jacob, 2016), a subject who weighs $75 \mathrm{~kg}$ would need to consume approximately $13-17 \mathrm{~g} / \mathrm{d}$ of shrimp oil to achieve similar benefits in glucose homeostasis observed rats with $15-20 \%$ dietary lard being replaced with shrimp oil. The higher dose should be considered for the better effect. However, this may be somewhat of an overestimate. For example, many of the beneficial effects of marine long-chain n-3 PUFA (e.g. EPA and DHA) occur at doses between 1-2 g/d whereas $17 \mathrm{~g}$ of shrimp oil would provide over 3 $\mathrm{g}$ of EPA and DHA.

\section{Conclusion}

Feeding of a high-fat diet and fructose in drinking water to rats induced rapidly obesity and insulin resistance. Replacement up to $20 \%$ of dietary lard with shrimp oil produced substantial improvements in oral glucose tolerance and insulin and leptin resistance, accompanied by the reductions of chronic inflammation and oxidative stress. Shrimp oil supplementation reversed reductions of the adiponectin and increases of free fatty acids in the blood, and decreased blood total cholesterol caused by high-fat diet and fructose-drinking water. These effects of shrimp oil supplementation occurred in the absence of a change in body weight. Shrimp oil is a promising functional food ingredient for the prevention and treatment of insulin resistance and type 2 diabetes.

\section{Conflict of Interest}

The authors declare that there is no conflict of interest. 


\section{Author Contributions}

J.G., J.Z, H.S.E, V.E and Y.W. conceived the rationale and objectives of the animal study; J.G., C.P, N.T, J.Z. and G.J. contributed shrimp oil, analytical methods and chemical analysis of shrimp oil. Y.W. designed and supervised the animal experiment. S.N. conducted the animal trial and laboratory analyses of samples. S.N. and Y.W. performed data analysis and manuscript preparation with all others contributing in data interpretation, discussion and editing.

\section{Acknowledgements}

The authors acknowledge financial support from the Atlantic Canada Opportunities Agency through the Atlantic Innovation Fund grant (\#193594) and from the Natural Health Products program with the Aquatic and Crop Resource Development Portfolio of National Research Council Canada. The authors thank all members of the Laboratory and Analytical Services team at the Coastal Zones Research Institute (CZRI) for their technical assistance. The authors are indebted to Island Fisherman Cooperative Association (IFCA) for providing the shrimp oil. The authors appreciate the assistance of Long Ma in the Department of Psychology and Neuroscience, Dalhousie University, Halifax, NS, Canada in animal necropsy and the support and care of animals provided by technicians in the animal facility of the Atlantic Veterinary College, University of Prince Edward Island, Charlottetown, PE, Canada over the course of the experiments.

\section{References}


Akiyama, T., Tachibana, I., Shirohara, H., Watanabe, N., and Otsuki, M. 1996. High-fat hypercaloric diet induces obesity, glucose intolerance and hyperlipidemia in normal adult male Wistar rat. Diabetes Res. Clin. Pract. 31(1-3): 27-35. PMID: 8792099.

Albert, B. B., Derraik, J. G. B., Brennan, C. M., Biggs, J. B., Smith, G. C., Garg, M. L., et al. 2014. Higher omega-3 index is associated with increased insulin sensitivity and more favourable metabolic profile in middle-aged overweight men. Sci. Rep. 4(2014): 6697. doi:10.1038/srep06697. PMID: 25331725.

Ambati, R. R., Phang, S. M., Ravi, S., and Aswathanarayana, R. G. 2014. Astaxanthin: sources, extraction, stability, biological activities and its commercial applications--a review. Mar. Drugs, 12(1): 128-152. doi:10.3390/md12010128. PMID:24402174.

Baik, I., Abbott, R. D., Curb, J. D., and Shin, C. 2010. Intake of fish and n-3 fatty acids and future risk of metabolic syndrome. J. Am. Diet. Assoc. 110(7): 1018-1026. doi:10.1016/j.jada.2010.04.013. PMID:20630158.

Considine, R. V., Sinha, M. K., Heiman, M. L., Kriauciunas, A., Stephens, T. W., Nyce, M. R., et al. 1996. Serum immunoreactive-leptin concentrations in normal-weight and obese humans. N. Engl. J. Med. 334(5): 292-295. doi:10.1056/NEJM199602013340503. PMID:8532024.

Cordero-Herrera, I., MartÃn, M. Ã. n., Goya, L., and Ramos, S. 2015. Cocoa intake ameliorates hepatic oxidative stress in young Zucker diabetic fatty rats. Food Res. Int. 69(2015): 194201. doi:10.1016/j.foodres.2014.12.039.

Del Campo, J. A., Rodriguez, H., Moreno, J., Vargas, M. A., Rivas, J., and Guerrero, M. G. 2004. Accumulation of astaxanthin and lutein in Chlorella zofingiensis (Chlorophyta). 
Appl. Microbiol. Biotechnol. 64(6): 848-854. doi:10.1007/s00253-003-1510-5. PMID:14689249.

Fassett, R. G., and Coombes, J. S. 2011. Astaxanthin: a potential therapeutic agent in cardiovascular disease. Mar. Drugs. 9(3): 447-465. doi:10.3390/md9030447. PMID:21556169.

Fernandez, M. L., and West, K. L. 2005. Mechanisms by which Dietary Fatty Acids Modulate Plasma Lipids1. J. Nutr. 135(9): 2075-2078. PMID:16140878.

Geng, S., Zhu, W., Xie, C., Li, X., Wu, J., Liang, Z., et al. 2016. Medium-chain triglyceride ameliorates insulin resistance and inflammation in high fat diet-induced obese mice. Eur. J. Nutr. 55(3): 931-940. doi:10.1007/s00394-015-0907-0. PMID:25911003.

Gray, B., Steyn, F., Davies, P. S., and Vitetta, L. 2013. Omega-3 fatty acids: a review of the effects on adiponectin and leptin and potential implications for obesity management. Eur. J. Clin. Nutr. 67(12): 1234-1242. doi:10.1038/ejen.2013.197. PMID:24129365.

Hill, J. O., Wyatt, H. R., and Peters, J. C. 2012. Energy balance and obesity. Circulation, 126(1): 126-132. doi:10.1161/CIRCULATIONAHA.111.087213. PMID:22753534.

Hosomi, R., Yoshida, M., and Fukunaga, K. 2012. Seafood consumption and components for health. Glob. J. Health Sci. 4(3): 72-86. doi:10.5539/gjhs.v4n3p72. PMID:22980234.

Huang, B. W., Chiang, M. T., Yao, H. T., and Chiang, W. 2004. The effect of high-fat and highfructose diets on glucose tolerance and plasma lipid and leptin levels in rats. Diabetes Obes. Metab. 6(2): 120-126. PMID:14746577.

Imamura, F., Micha, R., Wu, J. H., de Oliveira Otto, M. C., Otite, F. O., Abioye, A. I., et al. 2016. Effects of Saturated Fat, Polyunsaturated Fat, Monounsaturated Fat, and Carbohydrate on Glucose-Insulin Homeostasis: A Systematic Review and Meta-analysis 
of Randomised Controlled Feeding Trials. PLoS Med. 13(7): e1002087. doi:10.1371/journal.pmed.1002087. PMID:27434027.

Iwasaki, M., Hoshian, F., Tsuji, T., Hirose, N., Matsumoto, T., Kitatani, N., et al. 2012. Predicting efficacy of dipeptidyl peptidase- 4 inhibitors in patients with type 2 diabetes: Association of glycated hemoglobin reduction with serum eicosapentaenoic acid and docosahexaenoic acid levels. J. Diabetes Invest. 3(5): 464-467. doi:10.1111/j.20401124.2012.00214. PMID:24843607.

Jakicic, J. M. 2009. The effect of physical activity on body weight. Obesity (Silver Spring), 17 (Suppl 3): S34-38. doi:10.1038/oby.2009.386. PMID:19927144.

Johnson, A. R., Milner, J. J., and Makowski, L. 2012. The inflammation highway: metabolism accelerates inflammatory traffic in obesity. Immunol. Rev. 249(1): 218-238. doi:10.1111/j.1600-065X.2012.01151.x. PMID:22889225.

Jung, U. J., and Choi, M. S. 2014. Obesity and its metabolic complications: the role of adipokines and the relationship between obesity, inflammation, insulin resistance, dyslipidemia and nonalcoholic fatty liver disease. Int. J. Mol. Sci. 15(4): 6184-6223. doi:10.3390/ijms 15046184. PMID:24733068.

Kim, Y. S., Xun, P., and He, K. 2015. Fish consumption, long-chain omega-3 polyunsaturated fatty acid intake and risk of metabolic syndrome: a meta-analysis. Nutrients, 7(4): 20852100. doi:10.3390/nu7042085. PMID:25811108.

Koch, C. E., Lowe, C., Pretz, D., Steger, J., Williams, L. M., and Tups, A. 2014. High-fat diet induces leptin resistance in leptin-deficient mice. J. Neuroendocrinol. 26(2): 58-67. doi:10.1111/jne.12131. PMID:24382295. 
Konda, V. R., Desai, A., Darland, G., Grayson, N., and Bland, J. S. 2014. KDT501, a derivative from hops, normalizes glucose metabolism and body weight in rodent models of diabetes. PLoS One, 9(1): e87848. doi:10.1371/journal.pone.0087848. PMID:24498211.

Larsen, R., Eilertsen, K.-E., and Elvevoll, E. O. 2011. Health benefits of marine foods and ingredients. Biotechnol. Adv. 29(5): 508-518. doi:10.1016/j.biotechadv.2011.05.017. PMID: 21683131.

Lee, K. H., Ha, K. S., Jo, S. H., Lee, C. M., Kim, Y. C., Chung, K. H., et al. 2014. Effect of longterm dietary arginyl-fructose (AF) on hyperglycemia and HbAlc in diabetic $\mathrm{db} / \mathrm{db}$ mice. Int. J.

Mol. Sci. 15(5): 8352-8359. doi:10.3390/ijms15058352. PMID:24823880.

Li, X., Cai, X., Ma, X., Jing, L., Gu, J., Bao, L., et al. 2016. Short- and Long-Term Effects of Wholegrain Oat Intake on Weight Management and Glucolipid Metabolism in Overweight Type-2 Diabetics: A Randomized Control Trial. Nutrients, 8(9): 549. doi:10.3390/nu8090549. PMID:27618090.

Lombardo, Y. B., and Chicco, A. G. 2006. Effects of dietary polyunsaturated n-3 fatty acids on dyslipidemia and insulin resistance in rodents and humans. A review. J. Nutr. Biochem. 17(1): 1-13. doi:10.1016/j.jnutbio.2005.08.002. PMID:16214332.

Lorenzo, C., Wagenknecht, L. E., Hanley, A. J. G., Rewers, M. J., Karter, A. J., and Haffner, S. M. 2010. Hemoglobin A1c between 5.7 and $6.4 \%$ as a marker for identifying prediabetes, insulin sensitivity and secretion, and cardiovascular risk factors: The Insulin Resistance Atherosclerosis Study (IRAS). Diabetes Care, 33(9): 2104-2109. doi:10.2337/dc10-0679. PMID:20573754.

Madigan, C., Ryan, M., Owens, D., Collins, P., and Tomkin, G. H. 2000. Dietary unsaturated fatty acids in type 2 diabetes: higher levels of postprandial lipoprotein on a linoleic acid- 
rich sunflower oil diet compared with an oleic acid-rich olive oil diet. Diabetes Care, 23(10): 1472-1477. PMID:11023139.

Marques, C., Meireles, M., Norberto, S., Leite, J., Freitas, J., Pestana, D., et al. 2016. High-fat diet-induced obesity Rat model: a comparison between Wistar and Sprague-Dawley Rat. Adipocyte, 5(1): 11-21. doi:10.1080/21623945.2015.1061723. PMID:27144092.

Matthews, D. R., Hosker, J. P., Rudenski, A. S., Naylor, B. A., Treacher, D. F., and Turner, R. C. 1985. Homeostasis model assessment: insulin resistance and beta-cell function from fasting plasma glucose and insulin concentrations in man. Diabetologia, 28(7): 412-419. PMID:3899825.

Mauvais-Jarvis, F. 2015. Sex differences in metabolic homeostasis, diabetes, and obesity. Biol. Sex Differ. 6(2015): 14. doi:10.1186/s13293-015-0033-y. PMID:26339468.

Meydani, M., Natiello, F., Goldin, B., Free, N., Woods, M., Schaefer, E., et al. 1991. Effect of long-term fish oil supplementation on vitamin E status and lipid peroxidation in women. J. Nutr. 121(4): 484-491. PMID:1826131.

Mirmiran, P., Bahadoran, Z., and Azizi, F. 2014. Functional foods-based diet as a novel dietary approach for management of type 2 diabetes and its complications: A review. World J. Diabetes, 5(3): 267-281. doi:10.4239/wjd.v5.i3.267. PMID:24936248.

Nair, A. B., and Jacob, S. 2016. A simple practice guide for dose conversion between animals and human. J. Basic. Clin. Pharm. 7(2): 27-31. doi:10.4103/0976-0105.177703. PMID:27057123.

Nair, S. V., Zhang, J., and Wang, Y. 2014. Ethanol extract of Liuwei Dihuang reduces weight gain and visceral fat in obese-prone $\mathrm{CD}$ rats fed a high-fat diet. Exp. Biol. Med. (Maywood), 239(5): 552-558. doi:10.1177/1535370214525313. PMID:24603076 
Nettleton, J. A., and Katz, R. 2005. n-3 long-chain polyunsaturated fatty acids in type 2 diabetes: A review. J. Am. Diet. Assoc.. 105(3): 428-440. doi:10.1016/j.jada.2004.11.029. PMID:15746832.

Ohta, T., Katsuda, Y., Miyajima, K., Sasase, T., Kimura, S., Tong, B., et al. 2014. Gender differences in metabolic disorders and related diseases in Spontaneously Diabetic ToriiLepr(fa) rats. J. Diabetes Res. 2014(2014): 841957. doi:10.1155/2014/841957. PMID:24892034.

Patel, P. S., Sharp, S. J., Luben, R. N., Khaw, K. T., Bingham, S. A., Wareham, N. J., et al. 2009. Association between type of dietary fish and seafood intake and the risk of incident type 2 diabetes: the European prospective investigation of cancer (EPIC)-Norfolk cohort study. Diabetes Care, 32(10): 1857-1863. doi:10.2337/dc09-0116. PMID:19592633.

Popkin, B. M., Adair, L. S., and Ng, S. W. 2012. Global nutrition transition and the pandemic of obesity in developing countries. Nutr. Rev. 70(1): 3-21. doi:10.1111/j.17534887.2011.00456.x. PMID:22221213.

Pryor, W. A. 1991. The antioxidant nutrients and disease prevention--what do we know and what do we need to find out? Am. J. Clin. Nutr. 53(1 Suppl): 391S-393S. PMID:1985418.

Ramel, A., Martinéz, A., Kiely, M., Morais, G., Bandarra, N. M., and Thorsdottir, I. 2008. Beneficial effects of long-chain n-3 fatty acids included in an energy-restricted diet on insulin resistance in overweight and obese European young adults. Diabetologia, 51(7): 1261-1268. doi:10.1007/s00125-008-1035-7. PMID:18491071.

Riserus, U., Willett, W. C., and Hu, F. B. 2009. Dietary fats and prevention of type 2 diabetes. Prog. Lipid Res. 48(1): 44-51. doi:10.1016/j.plipres.2008.10.002. PMID:19032965. 
Rizvi, S., Raza, S. T., Ahmed, F., Ahmad, A., Abbas, S., and Mahdi, F. 2014. The Role of Vitamin E in Human Health and Some Diseases. Sultan Qaboos Univ. Med. J. 14(2): e157-e165. PMID:24790736.

Robbez Masson, V., Lucas, A., Gueugneau, A.-M., Macaire, J.-P., Paul, J.-L., Grynberg, A., et al. 2008. Long-Chain (n-3) Polyunsaturated Fatty Acids Prevent Metabolic and Vascular Disorders in Fructose-Fed Rats. J.Nutr. 138(10): 1915-1922. PMID:18806101.

Ross, R. 2003. Does exercise without weight loss improve insulin sensitivity? Diabetes Care, 26(3): 944-945. PMID:12610063.

Sabour, H., Norouzi Javidan, A., Latifi, S., Shidfar, F., Heshmat, R., Emami Razavi, S. H., et al. 2015. Omega-3 fatty acids' effect on leptin and adiponectin concentrations in patients with spinal cord injury: A double-blinded randomized clinical trial. J. Spinal Cord Med. 38(5): 599-606. doi:10.1179/2045772314Y.0000000251. PMID:25096818.

Sadighara, P., Saatloo, N. V., Omid, N. J., and Araghi, A. 2014. Shrimp Waste Oil: A Natural Supplement of Resistance Carotenoids Against Oxidation. Pharmacologia, 5(2014): 107109. doi:10.5567/pharmacologia.2014.107.109.

Samimi, M., Jamilian, M., Asemi, Z., and Esmaillzadeh, A. 2015. Effects of omega-3 fatty acid supplementation on insulin metabolism and lipid profiles in gestational diabetes: Randomized, double-blind, placebo-controlled trial. Clin. Nutr. 34(3): 388-393. doi:10.1016/j.clnu.2014.06.005. PMID:24973862.

Savini, I., Catani, M. V., Evangelista, D., Gasperi, V., and Avigliano, L. 2013. Obesityassociated oxidative stress: strategies finalized to improve redox state. Int. J. Mol. Sci. 14(5): 10497-10538. doi:10.3390/ijms140510497. PMID:23698776. 
Sila, A. d., Ghlissi, Z., Kamoun, Z., Makni, M., Nasri, M., Bougatef, A., et al. 2015. Astaxanthin from shrimp by-products ameliorates nephropathy in diabetic rats. Eur. J. Nutr. 54(2): 301-307. doi:10.1007/s00394-014-0711-2. PMID:24821271.

Singh, B., and Saxena, A. 2010. Surrogate markers of insulin resistance: A review. World J. Diabetes, 1(2): 36-47. doi: 10.4239/wjd.v1.i2.36. PMID:21537426.

Siriwardhana, N., Kalupahana, N. S., and Moustaid-Moussa, N. 2012. Health benefits of n-3 polyunsaturated fatty acids: eicosapentaenoic acid and docosahexaenoic acid. Adv. Food Nutr. Res. 65(2012): 211-222. doi:10.1016/b978-0-12-416003-3.00013-5. PMID:22361189.

Smit, M. J., Temmerman, A. M., Wolters, H., Kuipers, F., Beynen, A. C., and Vonk, R. J. 1991. Dietary fish oil-induced changes in intrahepatic cholesterol transport and bile acid synthesis in rats. J. Clin. Invest. 88(3): 943-951. doi:10.1172/jci115397. PMID:1885779.

Stranahan, A. M., Norman, E. D., Lee, K., Cutler, R. G., Telljohann, R. S., Egan, J. M., et al. 2008. Diet-induced insulin resistance impairs hippocampal synaptic plasticity and cognition in middle-aged rats. Hippocampus, 18(11): 1085-1088. doi 10.1002/hipo.20470. PMID:18651634.

Subramanian, B., Thibault, M. H., Djaoued, Y., Pelletier, C., Touaibia, M., and Tchoukanova, N. 2015. Chromatographic, NMR and vibrational spectroscopic investigations of astaxanthin esters: application to "Astaxanthin-rich shrimp oil" obtained from processing of Nordic shrimps. Analyst, 140(21): 7423-7433. doi:10.1039/c5an01261a. PMID:26393239.

Swanson, D., Block, R., and Mousa, S. A. 2012. Omega-3 Fatty Acids EPA and DHA: Health Benefits Throughout Life. Adv. Nutr. 3(1): 1-7. doi:10.3945/an.111.000893. PMID:22332096. 
Trebble, T., Arden, N. K., Stroud, M. A., Wootton, S. A., Burdge, G. C., Miles, E. A., et al. 2003. Inhibition of tumour necrosis factor-alpha and interleukin 6 production by mononuclear cells following dietary fish-oil supplementation in healthy men and response to antioxidant co-supplementation. Br. J. Nutr. 90(2): 405-412. PMID:12908901.

Valdes-Ramos, R., Guadarrama-Lopez, A. L., Martinez-Carrillo, B. E., and Benitez-Arciniega, A. D. 2015. Vitamins and type 2 diabetes mellitus. Endocr. Metab. Immune Disord. Drug Targets, 15(1): 54-63. PMID:25388747.

Vincent, H. K., Bourguignon, C. M., Weltman, A. L., Vincent, K. R., Barrett, E., Innes, K. E., et al. 2009. Effects of antioxidant supplementation on insulin sensitivity, endothelial adhesion molecules, and oxidative stress in normal-weight and overweight young adults. Metabolism, 58(2): 254-262. doi:10.1016/j.metabol.2008.09.022. PMID:19154960.

Wang, Y., Fofana, B., Roy, M., Ghose, K., Yao, X., Nixon, M., et al. 2015. Flaxseed lignan secoisolariciresinol diglucoside improves insulin sensitivity through upregulation of GLUT4 expression in diet-induced obese mice. J. Funct. Foods, 18(Part A): 1-9. doi:10.1016/j.jff.2015.06.053.

Wang, Y., Yi, X., Ghanam, K., Zhang, S., Zhao, T., and Zhu, X. 2014. Berberine decreases cholesterol levels in rats through multiple mechanisms, including inhibition of cholesterol absorption. Metabolism, 63(9): 1167-1177. doi:10.1016/j.metabol.2014.05.013. PMID:25002181.

Wellen, K. E., and Hotamisligil, G. S. 2005. Inflammation, stress, and diabetes. J. Clin. Invest. 115(5): 1111-1119. doi:10.1172/JCI25102. PMID: 15864338. 
Wilcox, G. 2005. Insulin and insulin resistance. Clin. Biochem. Rev. 26(2): 19-39. PMID:16278749.

Yamauchi, T., Kamon, J., Waki, H., Terauchi, Y., Kubota, N., Hara, K., et al. 2001. The fatderived hormone adiponectin reverses insulin resistance associated with both lipoatrophy and obesity. Nat. Med. 7(8): 941-946. doi:10.1038/90984. PMID:11479627.

Yanni, A. E., Stamataki, N. S., Konstantopoulos, P., Stoupaki, M., Abeliatis, A., Nikolakea, I., et al. 2016. Controlling type-2 diabetes by inclusion of Cr-enriched yeast bread in the daily dietary pattern: a randomized clinical trial. Eur. J. Nutr. in process. doi:10.1007/s00394016-1315-9. PMID:27734127.

Yoon, M. J., Lee, G. Y., Chung, J. J., Ahn, Y. H., Hong, S. H., and Kim, J. B. 2006. Adiponectin increases fatty acid oxidation in skeletal muscle cells by sequential activation of AMPactivated protein kinase, p38 mitogen-activated protein kinase, and peroxisome proliferator-activated receptor alpha. Diabetes, 55(9): 2562-2570. doi:10.2337/db051322. PMID:16936205.

Yuan, J. P., and Chen, F. 1997. Identification of astaxanthin isomers in Haematococcus lacustris by HPLC-photodiode array detection. Biotechnology Techniques 11(7): 455-459. doi: 10.1023/A:1018441411746. 
Table 1. Effect of shrimp oil supplementation on the body weight ( $\mathrm{g}$ ) of rats fed a high-fat diet and fructose-drinking water

\begin{tabular}{|c|c|c|c|c|c|c|c|c|c|c|c|}
\hline & \multicolumn{11}{|c|}{ Time post treatment (week) } \\
\hline & 0 & 1 & 2 & 3 & 4 & 5 & 6 & 7 & 8 & 9 & 10 \\
\hline LFC & $388.2 \pm 4.2$ & $447.2 \pm 6.0$ & $474.6 \pm 7.1$ & $506.3 \pm 7.7$ & $531.7 \pm 8.2$ & $558.3 \pm 8.7$ & $576.7 \pm 10.8$ & $598.4 \pm 10.8$ & $621.3 \pm 11.6$ & $636.0 \pm 12.0$ & $653.6 \pm 12.4$ \\
\hline $\mathrm{HFC}$ & $413.6 \pm 6.4$ & $478.9 \pm 8.6^{\dagger}$ & $509.5 \pm 9.9^{\dagger}$ & $552.0 \pm 10.6^{*}$ & $575.6 \pm 12.5^{\dagger}$ & $603.2 \pm 13.6^{\dagger}$ & $625.4 \pm 14.6^{*}$ & $651.8 \pm 15.2^{*}$ & $673.0 \pm 16.5^{*}$ & $694.2 \pm 16.9^{*}$ & $716.8 \pm 017.2^{*}$ \\
\hline SO10 & $407.0 \pm 4.9$ & $478.9 \pm 7.7$ & $514.8 \pm 9.5$ & $553.3 \pm 11.6$ & $580.6 \pm 14.2$ & $615.2 \pm 14.1$ & $647.0 \pm 16.5$ & $673.4 \pm 17.2$ & $698.9 \pm 17.9$ & $715.4 \pm 16.4$ & $733.1 \pm 17.6$ \\
\hline SO15 & $404.7 \pm 7.5$ & $480.2 \pm 8.5$ & $518.3 \pm 10.2$ & $563.7 \pm 11.9$ & $593.7 \pm 12.8$ & $624.4 \pm 14.0$ & $650.4 \pm 16.1$ & $677.0 \pm 16.2$ & $702.9 \pm 18.6$ & $723.4 \pm 18.3$ & $732.8 \pm 18.2$ \\
\hline $\mathrm{SO} 20$ & $406.0 \pm 4.2$ & $477.5 \pm 6.4$ & $512.2 \pm 8.0$ & $553.1 \pm 10.4$ & $580.0 \pm 11.8$ & $616.4 \pm 14.9$ & $642.7 \pm 16.2$ & $665.9 \pm 18.0$ & $685.62 \pm 19.5$ & $702.1 \pm 19.5$ & $715.7 \pm 21.8$ \\
\hline
\end{tabular}

The difference between the HFC and LFC groups was analyzed using repeated measures $t$-test. The treatment effect was analysed using repeated measures one-way ANOVA and differences among the HFC, SO10, SO15, and SO20 groups were determined using the least squares means test adjusted to Tukey. The significance least level was set to 0.05 . The results are presented as means \pm S.E.M ( $n=11-12) .{ }^{*} \mathrm{p}<0.05$ and ${ }^{\dagger} \mathrm{p}<0.01$ compared with LFC. 
Table 2. Effect of shrimp oil supplementation on the food intake (g) of rats fed a high-fat diet and fructose-drinking water

\begin{tabular}{|c|c|c|c|c|c|c|c|c|c|c|}
\hline & & & & & ime post tre & ment (week & & & & \\
\hline & 1 & 2 & 3 & 4 & 5 & 6 & 7 & 8 & 9 & 10 \\
\hline LFC & $30.5 \pm 0.7$ & $29.5 \pm 1.0$ & $28.8 \pm 1.1$ & $30.1 \pm 1.3$ & $28.3 \pm 0.8$ & $29.3 \pm 1.1$ & $28.0 \pm 0.6$ & $30.7 \pm 0.9$ & $29.2 \pm 0.8$ & $27.6 \pm 0.6$ \\
\hline HFC & $19.4 \pm 0.9^{\dagger}$ & $17.9 \pm 0.8^{\dagger}$ & $18.9 \pm 0.7^{*}$ & $18.7 \pm 0.7^{\dagger}$ & $17.6 \pm 0.7^{\dagger}$ & $17.1 \pm 0.7^{*}$ & $17.9 \pm 0.8^{*}$ & $17.1 \pm 0.8^{*}$ & $17.6 \pm 0.6^{*}$ & $19.6 \pm 0.5^{*}$ \\
\hline SO10 & $20.1 \pm 1.0$ & $19.2 \pm 1.1$ & $19.4 \pm 1.2$ & $19.4 \pm 1.2$ & $18.9 \pm 1.1$ & $19.4 \pm 1.1$ & $19.4 \pm 1.1$ & $17.3 \pm 0.1$ & $17.5 \pm 0.7$ & $18.0 \pm 0.2^{b}$ \\
\hline SO15 & $20.0 \pm 1.1$ & $20.0 \pm 1.0$ & $19.7 \pm 1.0$ & $19.9 \pm 0.9$ & $18.7 \pm 0.7$ & $18.7 \pm 0.9$ & $19.6 \pm 0.7$ & $17.3 \pm 0.3$ & $17.5 \pm 0.2$ & $18.0 \pm 0.2^{b}$ \\
\hline $\mathrm{SO} 20$ & $20.4 \pm 1.0$ & $19.8 \pm 0.8$ & $18.7 \pm 0.7$ & $18.8 \pm 0.5$ & $18.2 \pm 0.7$ & $18.2 \pm 0.6$ & $18.5 \pm 0.7$ & $17.2 \pm 0.2$ & $17.4 \pm 0.4$ & $17.9 \pm 0.3^{b}$ \\
\hline
\end{tabular}

The difference between the HFC and LFC groups was analyzed using repeated measures $t$-test. The treatment effect was analysed using repeated measures one-way ANOVA and differences among the HFC, SO10, SO15, and SO20 were determined using the least squares means test adjusted to Tukey. The significance least level was set to 0.05. The results are presented as means \pm S.E.M. $(n=$ 11-12). ${ }^{*} \mathrm{p}<0.05$ and ${ }^{\dagger} \mathrm{p}<0.01$ compared to LFC; ${ }^{\mathrm{p}}<0.01$ compared with HFC. 
Table 3. Effect of shrimp oil supplementation on semi-fasting blood glucose (mmol/l) of rats fed a high-fat diet and fructose-drinking water

\begin{tabular}{|c|c|c|c|c|c|c|c|c|c|c|c|}
\hline & \multicolumn{11}{|c|}{ Time post treatment (week) } \\
\hline & 0 & 1 & 2 & 3 & 4 & 5 & 6 & 7 & 8 & 9 & 10 \\
\hline LFC & $6.7 \pm 0.1$ & $6.6 \pm 0.1$ & $6.8 \pm 0.3$ & $6.7 \pm 0.2$ & $6.5 \pm 0.2$ & $6.4 \pm 0.2$ & $6.1 \pm 0.1$ & $6.1 \pm 0.1$ & $5.9 \pm 0.2$ & $6.1 \pm 0.2$ & $6.2 \pm 0.2$ \\
\hline $\mathrm{HFC}$ & $7.0 \pm 0.2$ & $7.0 \pm 0.2$ & $6.8 \pm 0.1$ & $7.1 \pm 0.2$ & $7.2 \pm 0.2$ & $6.9 \pm 0.2$ & $6.8 \pm 0.2$ & $6.9 \pm 0.2^{\dagger}$ & $6.7 \pm 0.1^{\dagger}$ & $6.9 \pm 0.1^{\dagger}$ & $7.0 \pm 0.2 *$ \\
\hline SO10 & $7.0 \pm 0.2$ & $6.9 \pm 0.2$ & $6.7 \pm 0.2$ & $7.0 \pm 0.1$ & $7.4 \pm 0.4$ & $6.9 \pm 0.2$ & $6.8 \pm 0.1$ & $6.9 \pm 0.1$ & $6.6 \pm 0.1$ & $6.8 \pm 0.2$ & $6.6 \pm 0.1$ \\
\hline SO15 & $6.9 \pm 0.2$ & $6.9 \pm 0.2$ & $6.7 \pm 0.2$ & $6.9 \pm 0.1$ & $7.2 \pm 0.2$ & $6.9 \pm 0.1$ & $6.8 \pm 0.1$ & $6.4 \pm 0.2$ & $6.8 \pm 0.2$ & $6.8 \pm 0.2$ & $6.6 \pm 0.1$ \\
\hline $\mathrm{SO} 20$ & $6.9 \pm 0.1$ & $6.7 \pm 0.1$ & $6.7 \pm 0.2$ & $6.8 \pm 0.2$ & $6.9 \pm 0.2$ & $6.9 \pm 0.1$ & $6.7 \pm 0.2$ & $6.5 \pm 0.1$ & $6.5 \pm 0.2$ & $6.7 \pm 0.2$ & $6.4 \pm 0.2$ \\
\hline
\end{tabular}

The difference between the HFC and LFC groups was analyzed using repeated measures $t$-test. The treatment effect was analysed using repeated measures one-way ANOVA and differences among the HFC, SO10, SO15, and SO20 were determined using the least squares means test adjusted to Tukey. The significance least level was set to 0.05. The results are presented as means \pm S.E.M. $(n=$ 11-12). ${ }^{*} \mathrm{p}<0.05$ and ${ }^{\dagger} \mathrm{p}<0.01$ compared to LFC. 
Table 4. Effect of shrimp oil supplementation on serum hemoglobin A1c, insulin, leptin, adiponectin, fasting glucose, lipids and insulin resistance index in high fat fed insulin resistant rats fed a high-fat diet and fructose-drinking water

\begin{tabular}{|c|c|c|c|c|c|}
\hline & LFC & HFC & SO10 & SO15 & $\mathrm{SO} 20$ \\
\hline $\begin{array}{l}\text { Fasting blood glucose } \\
\qquad(\mathrm{mmol} / \mathrm{l})\end{array}$ & $8.77 \pm 0.23$ & $9.30 \pm 0.33$ & $9.17 \pm 0.29$ & $8.76 \pm 0.18$ & $8.63 \pm 0.38$ \\
\hline Hemoglobin A1c (\%) & $4.0 \pm 0.1$ & $4.6 \pm 0.2 *$ & $4.1 \pm 0.1^{b}$ & $4.1 \pm 0.1^{\mathrm{b}}$ & $4.0 \pm 0.1^{\mathrm{c}}$ \\
\hline Insulin (ng/ml) & $0.59 \pm 0.04$ & $0.92 \pm 0.08^{\dagger}$ & $0.86 \pm 0.08$ & $0.67 \pm 0.10$ & $0.48 \pm 0.09^{b}$ \\
\hline Leptin (pg/ml) & $660.1 \pm 43.2$ & $1207.9 \pm 144.0^{\dagger}$ & $965.6 \pm 136.1$ & $950.8 \pm 124.3$ & $755.7 \pm 104.6^{\mathrm{a}}$ \\
\hline Adiponectin $(\mu \mathrm{g} / \mathrm{ml})$ & $10.2 \pm 0.6$ & $6.0 \pm 0.2^{*}$ & $8.6 \pm 0.7^{b}$ & $9.1 \pm 0.4^{\mathrm{c}}$ & $9.6 \pm 0.4^{\mathrm{d}}$ \\
\hline HOMA-IR & $5.1 \pm 0.4$ & $9.3 \pm 0.9^{*}$ & $7.8 \pm 0.9$ & $6.3 \pm 0.9$ & $4.7 \pm 0.5^{\mathrm{a}}$ \\
\hline $\begin{array}{l}\text { Free Fatty acids } \\
\qquad(\mathrm{mmol} / \mathrm{ml})\end{array}$ & $0.35 \pm 0.05$ & $0.72 \pm 0.13 *$ & $0.64 \pm 0.15$ & $0.53 \pm 0.09$ & $0.41 \pm 0.05$ \\
\hline Total cholesterol $(\mathrm{mg} / \mathrm{dl})$ & $118 \pm 9.4$ & $125.7 \pm 7.5$ & $114.7 \pm 9.2$ & $108.9 \pm 4.3$ & $94.4 \pm 7.9^{\mathrm{a}}$ \\
\hline HDL-cholesterol (mg/dl) & $66.9 \pm 5.1$ & $29.7 \pm 2.8^{\xi}$ & $28.5 \pm 1.9$ & $28.6 \pm 2.0$ & $26.0 \pm 2.0$ \\
\hline Triacylglycerols (mg/dl) & $195.6 \pm 22.4$ & $92.1 \pm 6.6^{\ddagger}$ & $88.6 \pm 9.5$ & $78.6 \pm 7.5$ & $77.0 \pm 8.4$ \\
\hline
\end{tabular}


The difference between the HFC and LFC was analyzed using paired student $t$-test. The treatment effect was analysed using one-way ANOVA and differences among the HFC, SO10, SO15, and SO20 were determined using the least squares means test adjusted to Tukey. The significance least level was set to 0.05 . The results are presented as means \pm S.E.M. $(n=11-12) .{ }^{*} p<0.05,{ }^{\dagger} p<0.01,{ }^{\dagger} p<$ $0.001,{ }^{\xi} p<0.0001$ compared to LFC; ${ }^{\mathrm{a}} \mathrm{p}<0.05,{ }^{\mathrm{b}} \mathrm{p}<0.01,{ }^{\mathrm{c}} \mathrm{p}<0.001$, and ${ }^{\mathrm{d}} \mathrm{p}<0.0001$ compared to HFC. 
Table 5. Effect of shrimp oil supplementation on the area under the curve (AUC) of blood glucose levels in the oral glucose tolerance and insulin tolerance tests in rats fed a high-fat diet and fructose-drinking water

\begin{tabular}{cccc}
\hline \multicolumn{3}{c}{ AUC of OGTT } & AUC of ITT \\
\hline & Week 4 & Week 9 & Week 10 \\
\hline LFC & $2232.1 \pm 49.8$ & $2258.2 \pm 52.2$ & $1493 \pm 58.4$ \\
HFC & $2552.6 \pm 22.0$ & $2663.1 \pm 53.9^{\ddagger}$ & $1847.6 \pm 49.7^{\ddagger}$ \\
SO10 & $2508.8 \pm 57.2$ & $2567.1 \pm 49.5$ & $1653.5 \pm 37.6^{\mathrm{a}}$ \\
SO15 & $2488.6 \pm 39.6$ & $2373.8 \pm 77.8^{\mathrm{a}}$ & $1583.5 \pm 38.2^{\mathrm{c}}$ \\
SO20 & $2373 \pm 38.1^{\mathrm{b}}$ & $2334.3 \pm 65.8^{\mathrm{b}}$ & $1540.9 \pm 45.3^{\mathrm{d}}$ \\
& & & \\
\hline
\end{tabular}

For the AUC of OGTT, the repeated measures $t$-test and repeated measures one-way ANOVA were used. For the AUC of ITT, difference between the HFC and LFC groups was analyzed using $t$-test and the treatment effect was analysed using one-way ANOVA. Differences among the HFC, SO10, SO15, and SO20 were determined using the least squares means test adjusted to Tukey. The significance least level was set to 0.05 . The results are presented as means \pm S.E.M. (n= 11-12). ${ }^{\ddagger} \mathrm{p}<0.001$ compared to LFC; ${ }^{\mathrm{a}} \mathrm{p}<0.05,{ }^{\mathrm{b}} \mathrm{p}<0.01,{ }^{\mathrm{c}} \mathrm{p}<0.001$, and ${ }^{\mathrm{d}} \mathrm{p}<0.0001$ compared to HFC. 
Table 6. Effect of shrimp oil supplementation on serum total antioxidants and proinflammatory biomarkers and liver antioxidant enzymes in rats fed a high-fat diet and fructose-drinking water

\begin{tabular}{|c|c|c|c|c|c|}
\hline & LFC & HFC & SO10 & SO15 & $\mathrm{SO} 20$ \\
\hline $\begin{array}{l}\text { Serum total } \\
\text { antioxidant in Trolox } \\
\text { equivalent }(\mathrm{mmol} / \mathrm{l})\end{array}$ & $1.61 \pm 0.07$ & $1.58 \pm 0.07$ & $1.66 \pm 0.08$ & $1.71 \pm 0.09$ & $1.87 \pm 0.04^{\mathrm{a}}$ \\
\hline $\begin{array}{l}\text { Liver glutathione } \\
\text { reductase activity } \\
(\mathrm{nmol} / \mathrm{min} / \mathrm{ml})\end{array}$ & $140.3 \pm 13$ & $144.3 \pm 8.1$ & $181.7 \pm 7.3$ & $177.0 \pm 11$ & $186.6 \pm 12^{\mathrm{a}}$ \\
\hline $\begin{array}{l}\text { Liver glutathione } \\
\text { peroxidase activity } \\
\text { (nmol/min } / \mathrm{ml})\end{array}$ & $139.0 \pm 7.0$ & $148.2 \pm 7.2$ & $161.7 \pm 7.2$ & $162.2 \pm 5.5$ & $168.1 \pm 8.0$ \\
\hline $\begin{array}{l}\text { Liver catalase activity } \\
\qquad(\mathrm{nmol} / \mathrm{min} / \mathrm{ml})\end{array}$ & $47.7 \pm 1.2$ & $44.9 \pm 2.0$ & $50.0 \pm 1.0$ & $50.8 \pm 1.2^{\mathrm{a}}$ & $51.9 \pm 1.3^{b}$ \\
\hline $\begin{array}{l}\text { Liver SOD activity } \\
\qquad(\mathrm{U} / \mathrm{ml})\end{array}$ & $3.1 \pm 0.1$ & $1.7 \pm 0.2$ & $2.3 \pm 0.2$ & $2.7 \pm 0.2^{\mathrm{a}}$ & $3.1 \pm 0.3^{b}$ \\
\hline $\begin{array}{l}\text { Liver TBARS } \\
\qquad(\mu \mathrm{mol} / 1)\end{array}$ & $18.1 \pm 0.9$ & $20.2 \pm 1.5$ & $19.5 \pm 1.4$ & $19.4 \pm 1.1$ & $18.4 \pm 0.7$ \\
\hline Liver GSH $(\mu \mathrm{mol} / 1)$ & $37.7 \pm 0.9$ & $35.7 \pm 0.5$ & $37.7 \pm 0.3^{\mathrm{a}}$ & $37.9 \pm 0.5^{\mathrm{a}}$ & $38.5 \pm 0.4^{\mathrm{c}}$ \\
\hline Liver GSSG $(\mu \mathrm{mol} / 1)$ & $13.5 \pm 1.4$ & $13.6 \pm 0.7$ & $12.7 \pm 0.9$ & $11.6 \pm 0.9$ & $11.5 \pm 0.6$ \\
\hline $\begin{array}{c}\text { Liver GSH:GSSG } \\
\text { ratio }\end{array}$ & $2.9 \pm 0.3$ & $3.0 \pm 0.2$ & $3.3 \pm 0.3$ & $3.5 \pm 0.3$ & $3.6 \pm 0.2$ \\
\hline
\end{tabular}




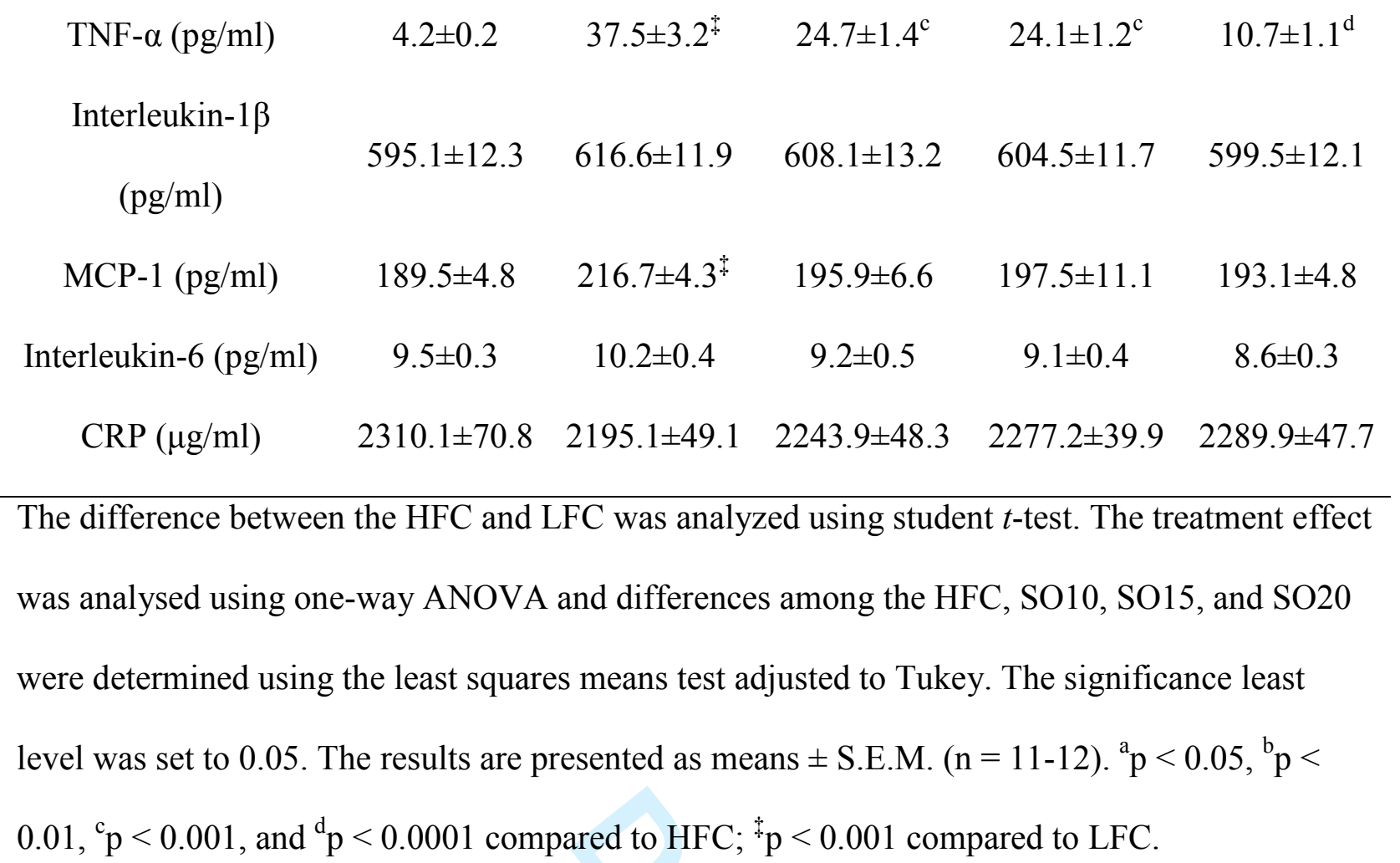


Figure 1. Effect of shrimp oil supplementation on oral glucose tolerance and insulin tolerance in rats fed a high-fat diet combined with fructose-drinking water. The difference between the HFC and LFC was analyzed using repeated measures $t$-test. The treatment effect was analyzed using one-way ANOVA with repeated measures and when a significant treatment effect was obtained, differences among the HFC, SO10, SO15 and SO20 were determined by pairwise comparisons using the least squares means test. Data are presented as means \pm S.E.M. $(n=11-12)$. A: the result of oral glucose tolerance test conducted in week 4 of the treatment. B: the results of oral glucose tolerance test conducted in week 9 of the treatment. ${ }^{*} \mathrm{p}<0.05,{ }^{\dagger} \mathrm{p}<0.01,{ }^{\dagger} \mathrm{p}<0.001$, and ${ }^{\#} \mathrm{p}<0.0001$ as compared to LFC; ${ }^{\mathrm{a}} \mathrm{p}<0.05,{ }^{\mathrm{b}} \mathrm{p}<0.01$, and ${ }^{\mathrm{c}} \mathrm{p}<0.001$ as compared to HFC.

Figure 2. Effect of shrimp oil supplementation on insulin tolerance in rats fed a high-fat diet combined with fructose-drinking water. The difference between the HFC and LFC was analyzed using repeated measures $t$-test. The treatment effect was analyzed using one-way ANOVA with repeated measures. When a significant treatment effect was obtained, differences among the HFC, SO10, SO15 and SO20 were determined by pairwise comparisons using the least squares means test. Data are presented as means \pm S.E.M. $(n=11-12) .{ }^{*} \mathrm{p}<0.05$ and ${ }^{\ddagger} \mathrm{p}<0.001$ as compared to LFC; ${ }^{a} p<0.05,{ }^{b} p<0.01$, and ${ }^{c} p<0.001$, as compared to HFC. 

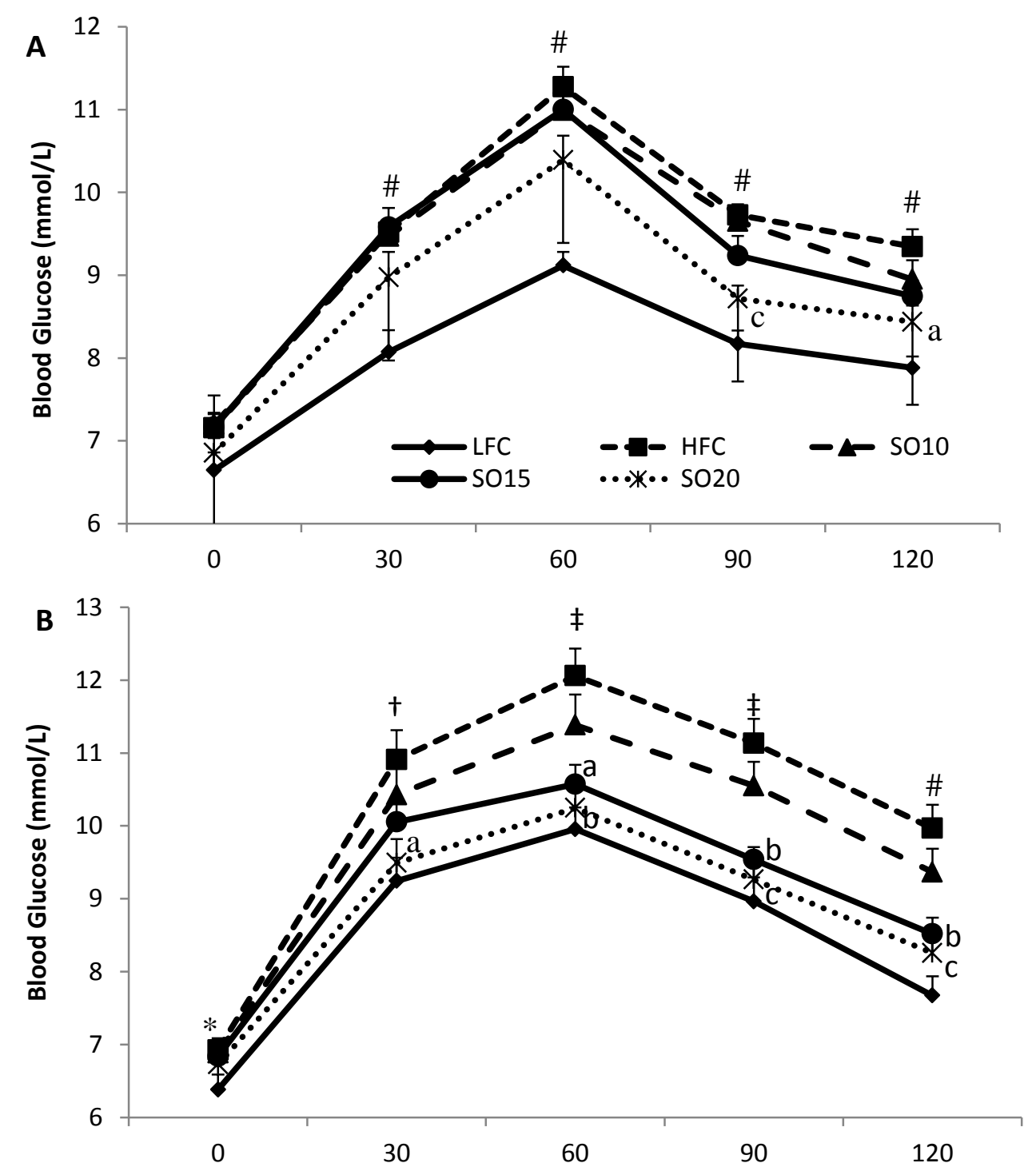

https://mc06.manuscriptcentral.com/apnm-pubs 


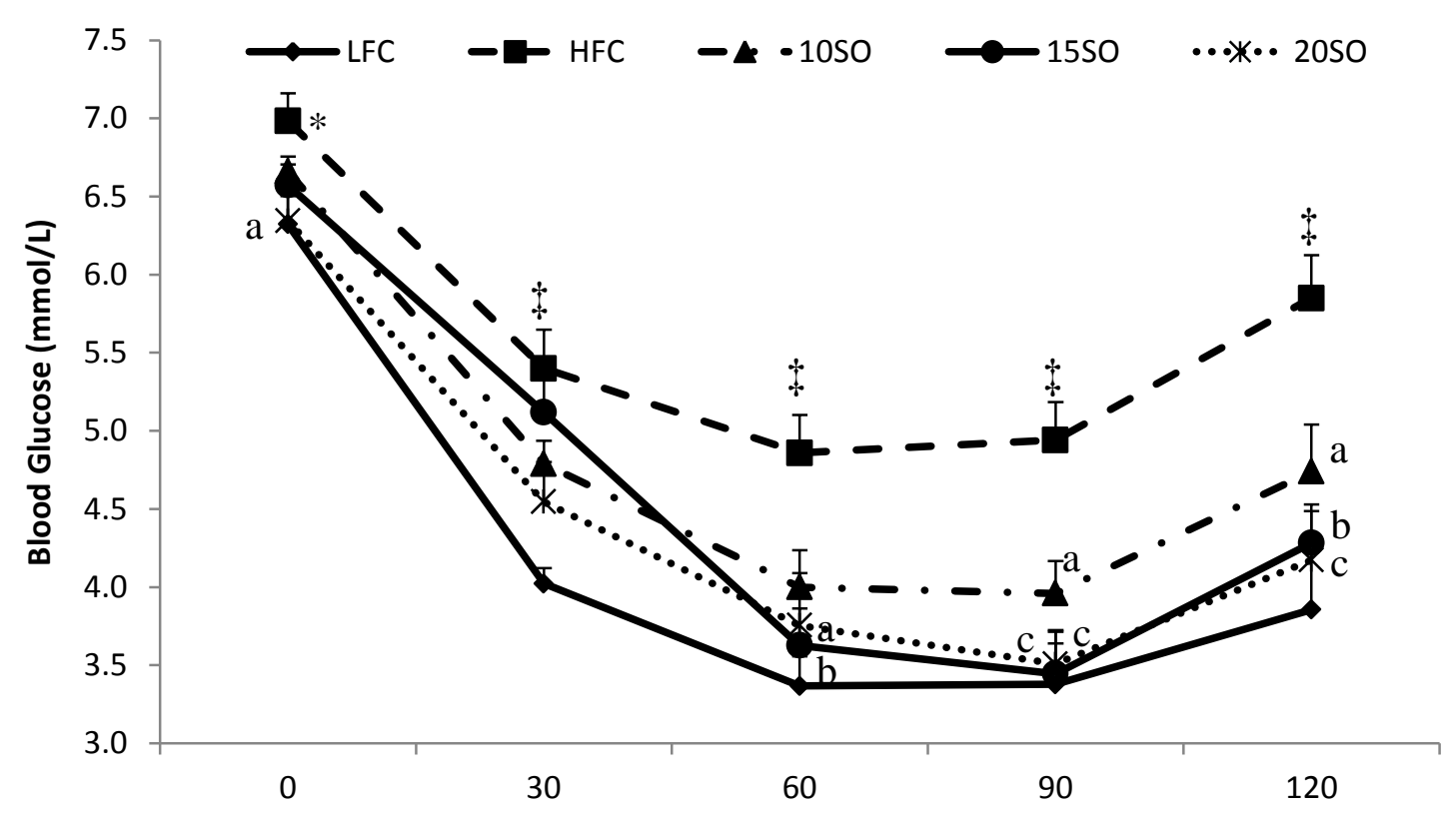

